\title{
ESTUDO COMPARATIVO E ANÁLISE INTEROBSERVADOR ENTRE DOIS MÉTODOS DE AVALIAÇÂO DA DISPLASIA COXOFEMORAL DE CÃES
}

\section{Comparative study and interobserver analysis between two diagnosis methods for hip dysplasia in dogs}

FROES, T.R. ${ }^{1}$; GARCIA, D.A.A. ${ }^{1}$; SCHMIDLIM, P.C. ${ }^{1}$; PARCHEN, H.D. ${ }^{1}$; SOUZA, A.C.R. ${ }^{1}$

1 Universidade Federal do Paraná, Curitiba/PR.

Endereço para correspondência: Tilde Rodrigues Froes: tilde@ufpr.br

\section{RESUMO}

Os objetivos desse trabalho foram avaliar as dificuldades da execução do método Penn HIP no diagnóstico da displasia coxofemoral, comparar essa modalidade com a empregada pelo CBRV e determinar a influência do treinamento na interpretação por meio de uma análise interobservador. Foi realizado o estudo radiográfico de 15 cães da raça Labrador, conforme as recomendações do CBRV e a análise do índice de distração articular. A comparação entre as técnicas se deu de forma subjetiva e pela concordância $K a p p a(K)$ quando na análise interobservador. Os valores de concordância para o método diagnóstico convencional foram $K=0,44$ (moderada) e pelo método PennHIP foi $K=1,0$ (quase perfeita). Todavia, houve maior variabilidade no PennHIP quando na comparação entre os membros. Conclui-se que o método PennHIP apresentou maior precisão diagnóstica e maior concordância interobservador para o diagnóstico da displasia coxofemoral bilateral em cães.

Palavras-chave: displasia pélvica canina; radiografia; variação dependente do observador

\section{ABSTRACT}

The aims of this study were to assess the difficulties of implementing PennHIP method in diagnosis of hip dysplasia, comparing this method with the recommended by CBRV and determining the influence of training in the interpretation through interobserver analysis. Fifteen dogs of the Labrador breed were used to evaluate the radiographic examination as the recommendations of CBRV and analysis of joint distraction. The agreement correlations of both methods were made by subjective and Kappa study. The values of conventional diagnostic method were $K=0.44$ (moderate) and almost perfect for PennHIP method $(K=1.0)$. However, there was higher variability in PennHIP when were compared both limbs. We concluded that the method PennHip showed greater diagnostic accuracy and higher interobserver agreement for the diagnosis of bilateral hip dysplasia in dogs.

Key words: hip dysplasia; observer variation; radiography 


\section{INTRODUÇÃO}

A displasia coxofemoral foi diagnosticada pela primeira vez por Schenelle em 1935, com o nome de luxação coxofemoral bilateral congênita (Riser, 1996). A displasia coxofemoral é uma doença poligênica de natureza quantitativa, multifatorial e extremamente complexa, que resulta em moléstia degenerativa da articulação coxofemoral. Além da hereditariedade e poligenia, a manifestação e desenvolvimento são dependentes de fatores intrínsecos e extrínsecos. Como exemplos destes fatores podemos citar: porte físico, estrutura e conformação corpórea, disparidade no desenvolvimento entre músculo e osso, taxa elevada de crescimento, nutrição excessiva e/ou inadequada, distrofia no músculo pectíneo, insuficiência da massa muscular pélvica, excesso de atividade física em cães jovens, manejo ambiental e aspectos hormonais, sendo a superalimentação e a raça fatores essenciais para sua manifestação (Lust et al., 1985; Alexander, 1992; Riser, 1996).

Algumas teorias sugerem que a displasia decorre de uma má formação da articulação coxofemoral, acompanhada de flacidez (lassidão) da cápsula articular (Smith et al., 1990; Alexander, 1992; Riser, 1996; Madsen, 1997), que se desenvolve durante o crescimento e a maturação do esqueleto, sendo estrutural e funcionalmente normal ao nascimento. Durante o crescimento, pode haver desequilíbrio entre o esqueleto e o sistema muscular de suporte que levam à incongruência articular. Ocorre uma disparidade no desenvolvimento dos tecidos ósseos e moles de suporte, que altera a biodinâmica da articulação, causando pequenos e repetidos traumas com conseqüente inflamação da membrana sinovial. O resultado dessas alterações será o surgimento de instabilidade articular e alterações ósseas degenerativas (Smith et al., 1990; Alexander, 1992; Riser, 1996; Madsen, 1997; Nogueira et al., 2005).

O diagnóstico pelo método radiográfico convencional (MCR) é realizado com objetivo de pré-seleção somente aos nove meses, embora cerca de $80 \%$ dos cães displásicos apresentem evidências de alterações radiográficas aos 12 meses e em alguns somente aos 24 meses (Lust et al., 1985; Brass, 1989; Normas, 2009). Segundo alguns pesquisadores, a idade mais recomendada para o diagnóstico confirmatório de displasia coxofemoral pelo método utilizado atualmente (MRC) é aos 24 meses, idade também preconizada pelo Colégio Brasileiro de Radiologia Veterinária (CBRV) (Nogueira et al, 2005; Normas, 2009).

Radiograficamente a displasia coxofemoral é caracterizada pelo arrasamento do acetábulo, alteração morfológica da cabeça do fêmur, subluxação ou luxação coxofemoral e outras alterações osteoartróticas secundárias (Lust et al., 1985; Riser, 1996; Allan, 2002).

Algumas técnicas auxiliares são utilizadas na avaliação radiográfica. Dentre elas, destaca-se a medida do ângulo de Norberg no filme radiográfico. Utilizando-se uma escala, mensura-se o ângulo formado por uma linha ideal que une as duas cabeças femorais e depois uma linha que une o centro da cabeça do fêmur sujeita a exame, com a borda acetabular craniolateral do mesmo lado. Qualquer medida constatada, inferior a $105^{\circ}$ mostra uma relação inadequada entre a cabeça do fêmur e o acetábulo, demonstrando sinais de subluxação ou luxação, o que pode ser caracterizado como displasia coxofemoral (Douglas e Williamson, 1975; Normas, 2009). Todavia, vale considerar que essa mensuração é questionada por alguns autores, devido principalmente a sua baixa especificidade (Culp et al., 2006). 
De qualquer forma, foi adotado pelo Colégio Brasileiro de Radiologia Veterinária (CBRV) a classificação em graus da displasia coxofemoral, sendo utilizado o ângulo de Norberg associado a demais características radiográficas, e por meio dessas, é estabelecida uma classificação: displasia leve ou discreta, displasia média ou moderada e displasia grave (Normas, 2009).

$\mathrm{Em}$ relação ao posicionamento conforme preconizado pelo MCR, evidências mostram que a extensão dos membros posteriores pode ocasionar torção dos tecidos moles da articulação, forçando a entrada da cabeça do fêmur no acetábulo, mascarando a presença de subluxação. Discute-se sobre esse fator como mais um dos determinantes da dificuldade de classificação desta enfermidade, sendo que a interferência do posicionamento associado a subjetividade da análise por diferentes observadores atue como fator perpetuante para a displasia (Heyman et al., 1993; Madsen, 1997).

O método radiográfico convencional confeccionado pela organização americana Orthopedic Foundation for Animals (OFA), similar ao utilizado pelo CBRV aparentemente é falho, principalmente na análise precoce, pois aproximadamente $55 \%$ dos cães não demonstram sinais de osteoartrose antes dos dois anos de idade. Isto pode dificultar a classificação, como supracitado, devido à subjetividade da análise da congruência articular entre a cabeça femoral e o acetábulo. Comprovou-se tal fato após confirmação de que cães inicialmente considerados negativos foram reclassificados como positivo quando idosos, sendo que nesse momento já apresentavam sinais de osteoartrose e displasia evidentes. Ou seja, a maioria dos animais com dois anos avaliados nos Estados Unidos da América que não apresentavam indução fenotípica 0 suficiente para a expressão da doença foram categorizados como negativos, apesar de serem portadores da doença (Smith e Gregor, 1993; Smith et al., 1997; Adams et al., 1998). Sabe-se que o diagnóstico positivo para a displasia é facilitado na presença de sinais secundários de doença articular degenerativa (Torres et al., 1999b; Adams, 2000; Nogueira et al., 2005).

Acredita-se que devido a subjetividade na análise dos filmes radiográficos, bem como as diferenças inter-observador fazem desse método utilizado pela OFA e CBRV pouco sensível (Smith et al., 1997; Adams et al., 1998). Há aproximadamente 20 anos essa modalidade de diagnóstico para displasia coxofemoral em cães é usada no Brasil e apesar de seleção altamente criteriosa e por diferentes radiologistas, não conseguiu-se observar a redução estatística da enfermidade. Isso demonstra pouca preocupação real no controle dessa enfermidade ou ineficiência no controle adotado (Torres et al., 1999a; Caldas, 2000; Torres et al., 2001; Urtado, 2005).

Em estudo recente realizado com trinta radiologistas, após análise interobservador quanto à leitura de filmes na projeção preconizada pela OFA, foi verificado que a repetibilidade da leitura dos filmes para a classificação entre cães displásicos e não displásicos foi de $72 \%$; Entretanto, essa repetibilidade foi somente de $43,6 \%$ para radiologistas inexperientes e $76 \%$ para os mais experientes quando se levou em conta diferentes graduações de displasia. Sendo assim, verifica-se que quanto menor a experiência profissional do radiologista maior a dificuldade em graduar a severidade da doença (Verhoeven et al., 2007).

Outra limitação dessa metodologia, atualmente aplicada no Brasil, é a faixa etária de 24 meses preconizada, uma vez que o início da idade reprodutiva de cães de grande porte está em torno de 10-12 meses (Normas, 2009). 
Devido à necessidade de se promover diagnósticos mais precoces diversos métodos têm sido pesquisados (Heyman et al., 1993; Smith e Gregor, 1993; Smith et al., 1997; Adams et al., 1998).

Visando então todos estes aspectos o Dr. Gail Smith da Universidade de Medicina Veterinária da Pensilvânia desenvolveu um método diagnóstico precoce para Displasia Coxofemoral, denominado PennHIP (Pennsylvania Hip Improvement Program) (Heyman et al., 1993; Smith e Gregor, 1993).

Após anos de estudo o pesquisador Gail Smith conseguiu provar teorias previamente muito discutidas, a principal dentre essas propostas é que o desenvolvimento da displasia coxofemoral está correlacionado à frouxidão da cápsula articular que sustenta essa articulação. 0 rápido crescimento em conjunto com o alto ganho de peso nos cães de grande porte iniciam uma série de eventos que levam a instabilidade e culminam no desenvolvimento da doença articular degenerativa ou osteoartrose (Smith et al., 1990).

Diferentes estruturas anatômicas são importantes para a manutenção da estabilidade articular dessa região, como o ligamento redondo (ligamento teres), a cápsula articular, a musculatura adjacente e a própria conformação da articulação. Sabe-se que quanto mais firme for a cápsula articular menor a chance de desenvolvimento da displasia (Riser, 1996; Nogueira et al., 2005).

A teoria da lassidão articular como um dos fatores mais importantes no desenvolvimento da displasia coxofemoral foi comprovada pelo método PennHIP (Riser, 1996; Smith et al., 1990; Smith e Gregor, 1993). Raças que apresentam uma perfeita coaptação articular, com uma cápsula articular firme e resistente não desenvolvem a doença, mesmo nas idades mais avançadas. Como exemplo destas raças pode citar, os Galgos (Smith et al., 1997).
Na tentativa de diminuir a também a subjetividade, e analisar realmente a flacidez articular foi desenvolvido um método quantitativo. Realizou-se um estudo radiográfico em estresse de compressão e distração por meio de um equipamento e obteve-se um índice de distração para diferentes raças. O método desenvolvido foi denominado Pennsylvania Hip Improvement Program - PennHIP. A pesquisa iniciou-se por volta de 1983 e desde 1996 vem sendo difundida pelos diferentes continentes. Recentes pesquisas comprovam a eficácia do método principalmente no que tange a reprodutibilidade e controle epidemiológico. Por esse método é possível também avaliar precocemente os animais com idade a partir de 16 semanas (Smith e Gregor, 1993; Madsen, 1997).

A identificação da laxidão articular é considerada um sinal precoce da displasia, e pelo MCR não é possível avaliá-la radiograficamente, pois esse dado só pode ser obtido pelas manobras de distração, sendo uma delas a técnica PennHIP, método no qual atualmente é o mais indicado para condução de análises de hereditariabilidade e precocidade diagnóstica (Adams et al., 1998; Nogueira et al., 2005).

O método PennHIP consiste na mensuração do índice de distração (ID). Este valor numérico é obtido mensurandose a distância do centro da cabeça femoral até 0 centro do acetábulo e depois dividindo-se este valor pela distância do raio formado pela cabeça femoral; se o resultado deste índice for menor ou igual a 0,3 o animal é considerado normal e se este índice for maior que 0,3 o animal é considerado displásico. Este resultado indica o quanto em porcentagem à cabeça femoral sai da junção. Por exemplo, com ID resultante em 0,75 significa que $75 \%$ da cabeça femoral concentra-se fora da junção (Smith et al., 1990; Allan et al., 2002). 
Considerando-se que a displasia coxofemoral é uma doença de alta ocorrência na população canina, e os animais acometidos apresentam grave diminuição na qualidade de vida quando acometidos, é importante escolhermos técnicas que proporcionem precocidade na elucidação diagnóstica, com a finalidade de promover um melhor controle epidemiológico (Allan, 2002; Nogueira et al., 2005).

Os objetivos do presente estudo foram avaliar as possíveis dificuldades da execução do método PennHIP, comparar a execução desse método com o rotineiramente empregado pelo CBRV nesses animais e determinar a influência do treinamento radiográfico na leitura dos filmes da região pélvica pelas duas técnicas.

\section{MATERIAL E MÉTODOS}

Foram utilizados 15 cães da raça Retriever do Labrador, sendo 08 machos e 07 fêmeas, com idade variando de oito a catorze meses (média de 12,6 meses), pesando entre 19 a $30 \mathrm{Kg}$ (média de peso de $23 \mathrm{Kg}$ ). Para a realização de todos os estudos radiográficos os animais foram anestesiados, com o seguinte o protocolo: medicação pré-anestésica, por via intramuscular associando acepromazina $(0,1 \mathrm{mg} / \mathrm{kg})$, diazepan $(0,5 \mathrm{mg} / \mathrm{kg})$, meperidina (5 $\mathrm{mg} / \mathrm{kg})$ e quetamina (3 $\mathrm{mg} / \mathrm{kg}$ ); tanto a indução quanto a manutenção anestésica,foram realizadas via intravenosa com propofol $(5 \mathrm{mg} / \mathrm{kg}$ ). Para efetuar a técnica de distração utilizouse o distrator (Figura 1).

Para realização do exame pelo método radiográfico convencional utilizaram-se as recomendações específicas do CBRV. No posicionamento para distração articular, realizou-se projeção em decúbito ventro-dorsal com o cão apoiado em calha de espuma, e três incidências radiográficas, a primeira projeção semelhante ao método convencional, a segunda fazendo-se a compressão dos fêmures sob acetábulo e a última projeção utilizando-se o distrator articular (Figura 2).

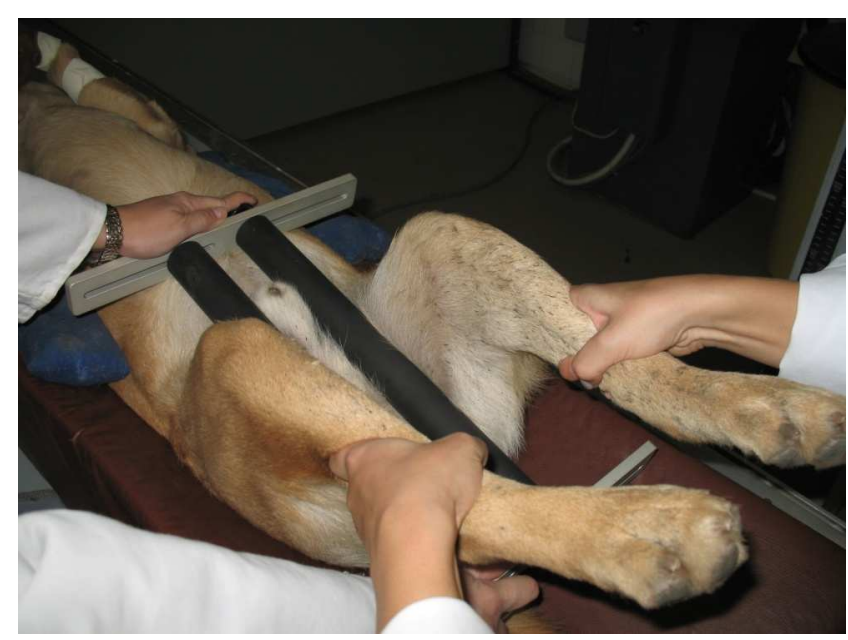

Figura 1 - Realização do exame com o distrator articular.

A finalidade da avaliação das radiografias obtidas pela técnica convencional (MRC) concentrou-se na busca de incongruência articular das cabeças femorais e sinais radiográficos sugestivos de doença articular degenerativa ou osteoartrose (presença de colar de osteófitos em cabeças femorais, linha de "Morgan", esclerose acetabular), além da mensuração do ângulo de Noberg. Para os filmes com o distrator, mensurouse o índice de distração (ID) conforme descrito em literatura (Smith et al., 1990; Smith e Gregor, 1993; Allan et al., 2002; Nogueira et al.,2005).

Para a obtenção do índice de distração (ID), colocou-se papel de seda sobre as radiografias, e utilizando-se 0 compasso determinou-se 0 centro do diâmetro da cabeça do fêmur na radiografia sem tração; confirmou-se a correta localização do centro do diâmetro na radiografia realizada com a distração e depois mensurou-se a distância até a borda do acetábulo; com a utilização de réguas e compasso, os valores do diâmetro da cabeça do fêmur e da sua distância até a borda do acetábulo foram determinadas. $O$ cálculo do ID foi resultante da divisão entre as duas mensu- 


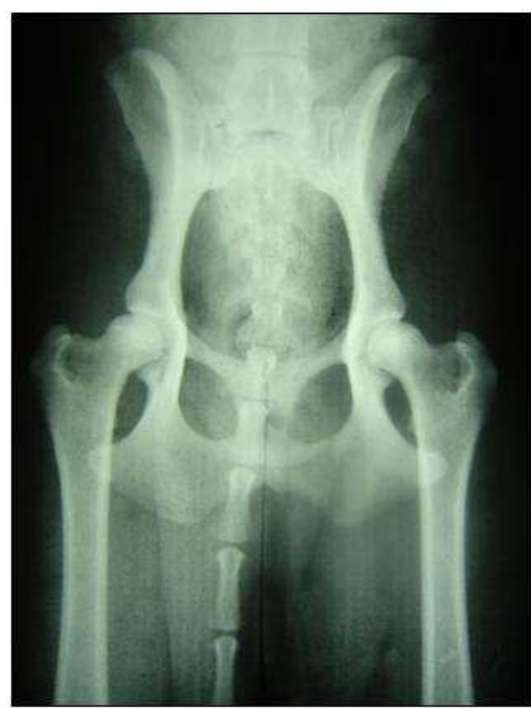

A

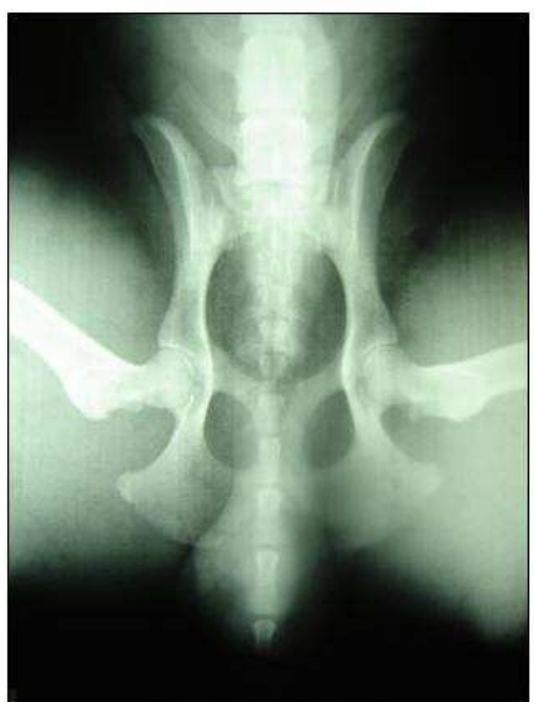

B

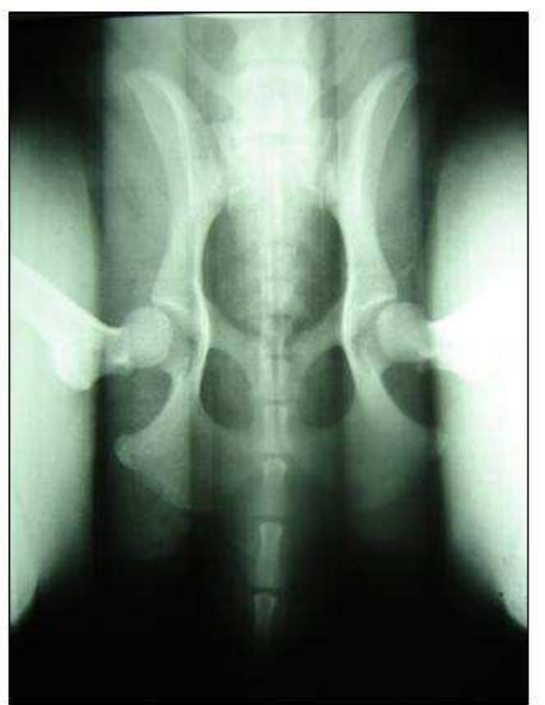

C

Figura 2 - A: Imagem radiográfica em projeção ventro-dorsal, técnica convencional; B: Imagem da radiográfica demonstrando a compressão dos fêmores sobre o acetábulo (técnica de compressão); C: Imagem radiográfica utilizando o distrator articular sobre a articulação coxofemoral (técnica distração).

rações previamente obtidas, determinando-se assim, a presença ou não da displasia pelos dois métodos.

Verificou-se a variabilidade de diagnóstico entre os radiologistas pelo método estatístico kappa (Siegel e Castellan, 1988) para estimar a substancial concordância entre os mesmos e a significância dos resultados obtidos. Essa análise foi feita em dois tempos. Em um primeiro momento para determinar a variabilidade observacional pelo método PennHIP na identificação da presença ou ausência da displasia, utilizando-se o valor 0,3 como a medida de corte. Em um segundo momento, analisou-se a variância interobservador quanto à presença ou ausência de displasia pelo método radiográfico convencional. $\mathrm{O}$ observador 1 era menos experiente estudando e atuando em diagnóstico por imagem há 2 anos, o observador 2 era 0 mais experiente atuando na especialidade há mais de 10 anos.

\section{RESULTADOS}

Pela técnica convencional (MRC) verificou-se que o observador 1 detectou a enfermidade em 12 animais (80\%), bilateralmente. E o observador 2 detectou a presença da displasia em $14(93,3 \%)$ dos animais examinados (Figura 3 ). Ainda pelo MCR analisando-se o ângulo de Norberg observou-se a seguinte distribuição em ambos os observadores: cães normais 3 $(20 \%)$, suspeitos $2(13,3 \%)$, displasia leve $9(60 \%)$, displasia média $1(6,7 \%)$ (Figura $4)$.

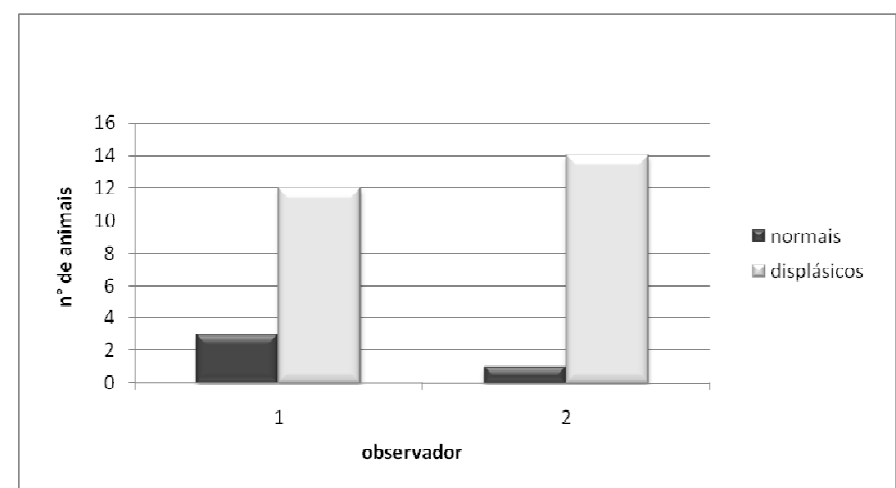

Figura 3 - Detecção de displasia através da avaliação das alterações subjetivas (MCR). 


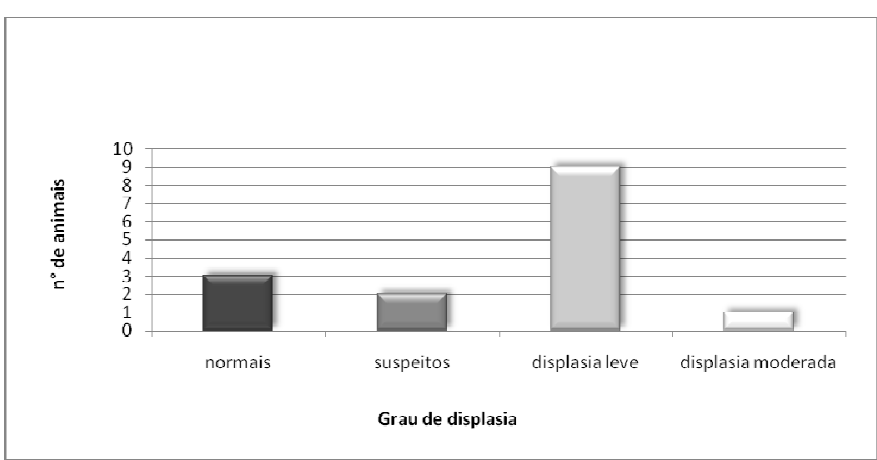

Figura 4 - Distribuição de ocorrência dos diversos graus de displasia avaliando-se apenas o ângulo de Norberg.

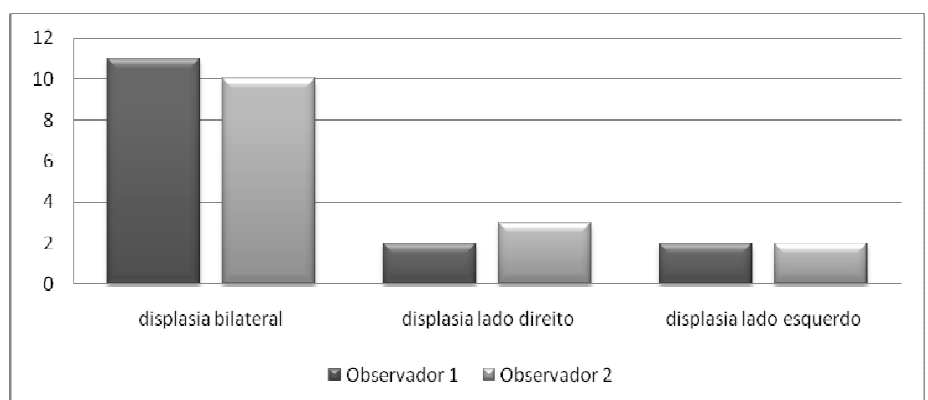

Figura 5 - Distribuição do diagnóstico de displasia segundo o índice de distração - PennHIP.

Pelo método PennHIP todos os 15 animais (100\%) radiografados apresentaram displasia coxofemoral, ou seja, o índice de distração era maior que 0,3 para ambos os observadores. Destes 11 cães $(73,3 \%)$ apresentavam displasia coxofemoral bilateralmente, 2 cães $(13,3 \%)$ tinham displasia unilateral do lado direito e outros 2 cães $(13,3 \%)$ apresentavam displasia unilateral do lado esquerdo, segundo o observador 1. Já para o observador 2, verificou-se que em 10 cães $(66,7 \%)$ haviam alterações bilaterais, em 3 cães (20\%) observou-se displasia unilateral do lado direito e 2 cães $(13,3 \%)$ detectou-se displasia unilateral do lado esquerdo. Portanto, se considerarmos a identificação da displasia de forma unilateral, pelo método PennHIP, o observador 1 confirmou a displasia unilateral em 4 animais, e o observador 2 confirmou a displasia unilateral em 5 animais (Figura 5).

A reprodutibilidade entre os observadores pelo método tradicional apresentou um valor $K$ (kappa) de 0,44 significando moderada concordância
(Siegel e Castellan, 1988) enquanto que pelo método PennHIP obteve-se um valor $K=1,0$ ou seja, uma concordância quase perfeita (Siegel e Castellan, 1988). Entretanto, como previamente citado houve maior variabilidade quando se considerou a identificação de presença ou ausência comparando-se paralelamente os dois membros, ou seja, ocorreu discordâncias entre os observadores na real identificação de qual o membro acometido quando na análise dos valores de distração, proporcionados pelo PennHIP. A concordância na identificação da presença ou não de displasia somente no membro esquerdo pelo método PennHIP foi igual a $K=0,423$ significando concordância moderada (Siegel e Castellan, 1988); para o membro direito $K=0,286$ significando leve concordância (Siegel e Castellan, 1988).

Devemos ressaltar ainda dentro dos resultados as dificuldades encontradas para a realização dos estudos radiográficos. Durante 0 posicionamento pelo MCR semelhante ao preconizado pelo CBRV, em alguns animais a tração se fez mais difícil, devido ao porte do animal. Para o método PennHIP exige-se um maior número de pessoas envolvidas durante 0 posicionamento, e sem dúvida uma maior força do executor na tração contra o equipamento. Assim, em cães mais pesados, principalmente machos, 0 procedimento se tornou mais difícil. Verificou-se uma evidente relação proporcional de força necessária para o posicionamento versus o tamanho do animal.

Verificou-se que o observador 1 , em treinamento, apresentou maior dificuldade em identificar a presença de sinais iniciais de doença articular degenerativa pelo MCR. Independente do grau de treinamento do radiologista, uma das maiores limitações foi a determinação exata do centro da cabeça do fêmur provavelmente devido à sobreposição dos tecidos moles adjacentes. A identificação exata da borda acetabular também pode 
ser de difícil determinação. Como alternativa, percebeu-se que uma melhor resolução dos limites da imagem ao se riscar um papel de seda sobre o filme, e após isso proceder a mensuração com mais exatidão.

\section{DISCUSSÃO}

A displasia coxofemoral é uma doença que ocasiona desconforto e dor nos cães levando a redução da longevidade e da qualidade de vida. $O$ diagnóstico da displasia coxofemoral tem grande importância na medicina veterinária uma vez que os tratamentos médicos e cirúrgicos não promovem uma cura efetiva, pois são técnicas paliativas preconizadas na tentativa de melhorar a qualidade de vida do paciente (Lust et al., 1985; River, 1996).

A literatura descreve diferentes métodos que têm sido utilizados para o diagnóstico precoce da displasia, métodos estes muito conceituados e tradicionais, entretanto, como exposto por vários pesquisadores estas técnicas apresentam algumas limitações metodológicas (Lust et al., 1985; Smith et al., 1997; Nogueira et al., 2005). Além disso, há mais de 30 anos discute-se sobre tais análises e verifica-se que a ocorrência nacional desta doença não tem reduzido (Torres et al., 1999a; Caldas, 2000; Torres et al., 2001; Urtado, 2005). Apesar do pequeno número de amostra desse experimento, pode-se inferir o elevado grau de ocorrência desta doença na raça Labrador, reafirmando a importância do controle da mesma. Esses dados nos fazem refletir também sobre a real contribuição da metodologia utilizada até o momento preconizada pelo CBRV para o controle da doença concordando com outros trabalhos publicados na literatura (Smith et al., 1990; Smith et al., 1997; Normas 2009). Precisamos nos atualizar no sentido de proporcionar melhores alternativas aos nossos animais, principalmente aqueles com potencial reprodutivo.

Com esse estudo notou-se que MCR somente três cães seriam considerados aptos a reprodução pelo observador $1 \mathrm{e}$ um cão pelo observador 2, contudo pelo método PennHIP, nenhum dos animais seriam aptos a reprodução. Assim se não fosse realizado o estudo por esta última metodologia, estes cães iriam contribuir para a perpetuação da doença em seus descendentes.

Com relação aos resultados de concordância pode-se notar que o Kappa foi considerado moderado quando na avaliação pelo MCR no diagnóstico da displasia, ou seja, o observador 1 (menos experiente) conseguiu detectar a presença da displasia em $80 \%$ dos animais, e observador 2 (mais experiente) conseguiu detectar a presença da displasia em 93,3\%. Ressalta-se que as lesões secundárias relativas a doença articular degenerativa, mesmo que tênues auxiliaram tal diagnóstico (Powers et al., 2004; 2007; Szabo et al., 2007).

Pelos dados obtidos, verificou-se que os novos radiologistas apresentaram uma maior dificuldade em identificar a incongruência articular, bem como, os primeiros sinais radiográficos da doença articular degenerativa. Estes dados foram semelhantes a outros estudos realizados inter-observadores (Verhoeven et al., 2007). Vale mais uma vez ressaltar que esses sinais precoces de doença articular degenerativa, como colar de osteófitos, osteófitos na borda dorsal do acetábulo e presença da linha de Morgan (Adams, 2000; Verhoeven et al., 2007), facilitam a identificação positiva da enfermidade ao MCR. Contudo, sugere-se que sejam necessários a realização de mais estudos nacionais com um maior número de pacientes e leitores a fim de obtermos dados mais consistentes sobre essa perspectiva. Afinal, sabe-se da subjetividade do exame radiográfico como modalidade diagnóstica entre diferentes 
interpretadores quando analisamos a estrutura pélvica, assim como em outras regiões anatômicas (Torres et al., 1999b; Gadbois et al., 2009), além disso, a literatura ainda afirma que o grau de treinamento tem grande influência na interpretação dos achados (Ricciardi, 2007).

O método PennHIP e outras técnicas de distração têm como principais características, além da detecção precoce da doença, prever alterações fenotípicas futuras; sendo assim modalidade ideal a ser utilizada no atual controle da displasia (Smih et al., 1990; Heyman et al., 1993; Allan, 2002). Aparentemente é uma das metodologias descritas na literatura que possui maior reprodutibilidade, uma vez que se trata da obtenção de um valor numérico reduzindo substancialmente a subjetividade diagnóstica. Os resultados desta pesquisa apresentam concordância com a literatura quanto a reprodutividade do método, afinal para o PennHip a concordância do Kappa foi $K=1$, considerada excelente na diferenciação entre cães displásicos e não displásicos. No entanto, percebeu-se que houve pouca concordância para a detecção da displasia unilateral, o que nos sugere pensar que mesmo sendo uma análise numérica, pode haver variações, e a sua execução não é tão simples assim. Programas de computação estão sendo criados com o intuito de melhorar ainda mais a realização das mensurações, e apresentam resultados promissores, principalmente com a introdução da radiologia computadorizada e digital (Ginga et al., 2006). Mesmo um método aparentemente mais preciso não estará isento de erros quando realizado por diferentes interpretadores.

Um dos fatores que provavelmente influenciaram na variação dos resultados de cálculo do ID pelo PennHIP estão correlacionadas a dificuldade de identificação do ponto diametral da cabeça femoral, como previamente mencionado.
Essas variações poderiam ser suprimidas com a utilização de um programa de computador, como alguns estudos já comprovam melhora nas correlações e na reprodutibilidade do método (Nogueira et al., 2005; Ginga et al., 2006).

A despeito das grandes vantagens do método PennHIP, um dos fatores pouco salientados na literatura é a dificuldade de tração do membro quando na realização das radiografias com o distrator. Percebeuse uma grande dificuldade de se tracionar os cães com maior aporte muscular, exigindo considerável força muscular do executor. Provavelmente, o grau de força ao se promover a distração articular proporcionado pelo executor da técnica pode interferir na medida final do ID, por isso um treinamento para execução da manobra de tração também é essencial, e mesmo assim ainda passível de erros. A força de tração deve ser perfeita e representada pela redução da linha radioluscente de ar identificada lateralmente ao equipamento, que corresponde o tocar da musculatura femoral sobre a borracha do equipamento (Smith e Gregor, 1993).

Quando analisamos a presença da displasia somente pelo ângulo de Norberg, cinco labradores seriam aptos à reprodução, três destes por terem um ângulo maior que $105^{\circ} \mathrm{e}$ dois por terem um ângulo igual a $105^{\circ}$. Porém estes resultados não são concordantes quando comparamos os resultados obtidos com a técnica PennHIP. Discordando com o mencionado por outros pesquisadores, que descrevem a possibilidade de identificação precoce de cães displásicos somente por meio do ângulo de Noberg (Culp et al., 2006), verificou-se que essa medida fornece algumas informações, entretanto, apresenta falhas quando comparada a outros métodos. Assim, sugere-se que não seja utilizada como técnica única de classificação, fato esse também afirmado pelo CBRV (Normas, 2009). 
Vale considerar que existem algumas vantagens do MCR exigido pelo CBRV, sejam elas: perfeita avaliação fenotípica da articulação, indicando os sinais de doença articular degenerativa (não proporcionada pelas técnicas de distração), e a necessidade de realizar somente uma única incidência radiográfica (Caldas, 2000; Torres et al., 1999b). Sua grande desvantagem em relação às inferências de distração está na impossibilidade de avaliar o grau de frouxidão articular, não podendo então ser considerada uma modalidade ideal para um controle indicador de cruzamentos com intuito de reduzir a incidência da enfermidade. Para esta finalidade, a efetividade na desclassificação deve ser rígida, e mesmo assim, há duvidas sobre seu eventual aproveitamento (Smith, 1997; Adams et al., 1998). Todavia, nunca devemos desconsiderar a utilização dessa incidência até porque a mesma deve ser realizada previamente as técnicas de distração, para se ter certeza de sua necessidade, já que muitos cães, mesmo jovens, apresentam evidências da enfermidade pelos achados indiretos de osteoartrose (Allan, 2002; Normas 2009).

\section{CONCLUSÕES}

O método PennHIP apresentou maior precisão diagnóstica e maior concordância interobservador para o diagnóstico da displasia coxofemoral bilateral em cães.

Deve-se utilizar o método na rotina para identificação e classificação da displasia coxofemoral no plantel nacional, como alternativa de precocidade diagnóstica, e ainda nos cães que são considerados suspeitos pelo método radiográfico convencional.

\section{AGRADECIMENTOS}

Ao Conselho Nacional de desenvolvimento Científico e Tecnológico (CNPq).

\section{REFERÊNCIAS}

ADAMS, W.A; DUELAND, R.T; MEINEX, J. et al. Early detection of canine Hip dysplasia: Comparison of two palpation and five radiographic methods. Journal of the American Animal Hospital Association, v.34, n.4 p.339-347, 1998.

ADAMS, W.M. Radiographic diagnosis of hip dysplasia in the young dog. Veterinary Clinics of North America: Small Animal Practice, v.30, n.2, p.267-280, 2000.

ALEXANDER, J.W. The pathogenesis of canine hip dysplasia. The Veterinary Clinics of North America: Small Animal Practice, v.22, n.3,p.503511, 1992.

ALLAN, G. Radiographic Signs of Joint Diasease In: THRALL, D. E. Textbook of Veterinary Diagnostic Radiology, 4. ed., cap. 16, p. 187-207, 2002.

ASSOCIAÇÃO BRASILEIRA DE RADIOLOGIA VETERINÁRIA. Normas da F.C.I. para avaliação da displasia coxofemoral. Disponível em: <http://www.abrv.com.br/col_normas.php > Acesso em 19/02/2009.

BRASS, W. Hip dysplasia in a dog. Journal of the small animal practice. V.30, n.3, p. 166-170, 1989.

CALDAS, E.L.C. Incidência da dysplasia coxofemoral em cães da raça Rottweiler Avaliação radiográfica. 2000. 45p. Dissertação (Mestrado em Clínica Médica) - Universidade Federal de Pernambuco, Recife.

CULP, W.T.N.; KAPATKIN, A. S.; GREGOR, T. P. et al. Evaluation of the Norberg angle threshold: a comparison of Norberg angle and distraction index as measures of coxofemoral degenerative joint disease susceptibility in seven breeds of dogs. Veterinary Surgery, v.35, n.5, p.453-9, 2006.

DOUGLAS, S.W.; WILLIAMSON, H.D. Diagnóstico radiológico veterinário. In: Diagnóstico radiológico veterinário. Zaragoza: Acribia, 1975, 330p.

GADBOIS, J.; D'ANJOU, M.A.; DUNN, M. et al. Radiographic abnormalities in cats with feline bronchial disease and intra - and interobserver variability in radiographic interpretation: 40 cases (1999-2006). Journal of American Veterinary Medical Association, v.234, n.3, p.367-375, 2009.

GINGA,M.M.; FERREIRA, A.J.; SILVESTRE, M. et al. Repeatability and reproducibility of distraction indices in PennHIP examinations of the hip joint in dogs. Acta veterinaria Hungarica, v.54, n.3, p.387-392, 2006. 
HEYMAN, J.; SMITH, G.K.; COFONE, M.A. A biomechanical study of the effect of coxofemoral positioning on passive hip joint laxity in the dog. American Journal of Veterinary Research, v.54, n.2, p.210-215, 1993.

LUST, G; RENDANO, V.T.; SUMMERS, B.A. Canine hip dysplasia: concepts and diagnosis. Journal of the America Veterinary Medical Association, v.187, n.6, p.638-40, 1985.

MADSEN, J.S. The joint capsule and joint laxity in dogs with hip dysplasia. Journal of the America Veterinary Medical Association, v.210, n.10, p.1463-5, 1997.

NOGUEIRA, S.R.; ROCHA, L.B.; TUDURY, E.A. Utilização do índice de distração no diagnostico da displasia coxofemoral canina. Clínica Veterinária, v.10, n.54, p.28-42, 2005.

POWERS, M.Y.; BIERY, D.N.; LAWLER, D.E. et al. Use of the caudolateral curvilinear osteophyte as an early maker for future development of osteoarthritis associated with hip dysplasia in dogs. Journal of American Veterinay Medical Association, v.225, n.2, p.233-237, 2004.

RICCIARDI, M. F. Concordância interobservador na avalição radiográfica torácica de cadelas com neoplasias mamárias. 2007. Curitiba, 169f. Dissertação (Mestrado em Ciências Veterinárias) - Curso de Pós-graduação em Ciências Veterinárias, Universidade Federal do Paraná.

RISER, W.H. Displasia coxofemoral canina In: BOJRAD, M.J. Mecanismos da moléstia na cirurgia de Pequenos Animais, 1 ed., Manole, 1996, p.924-932.

SIEGEL S, CASTELLAN N. Nonparametric Statistics for the Behavioral Sciences. 2.ed. New York: McGraw-Hill, 1988. p 284-285.

SMITH, G.K.; BIERY, D.N.; GREGOR, T.P. News concepts of coxofemoral joint stability and the clinical stress-radiographic method for quantificating hip joint laxity in the dog. Journal of the American Veterinary Medical Association, v.196, n.1, p. 59-70, 1990.

SMITH, G.K.; GREGOR, T.P. PennHIP Handbook. University of Pennsylvania Press, Philadelphia, PA, 1993, 102p.
SMITH, G.K.;LAFOND; GREGOR, T.P. Within-and between-examiner repeatability by experienced and inexperienced examiners in performing distraction stress-radiography to quantitate canine coxofemoral joint laxity. American Journal of Veterinay Research, v.58, n.10, p.1076-7, 1997.

SMITH, G.K. Advances in diagnosing canine hip dysplasia. Journal of American Veterinary Medical Association, v.210, n.10, p.1451-7, 1997.

SZABO, S.D.; BIERY, D.N.; LAWLER, D.F. et al. Evaliation of circumferential femoral head osteophyte as an early indicator of osteoarthritis characteristic of canine hip dysplasia in dogs. Journal of American Veterinary Medical Association, v.213, n.6, p.889-892, 2007.

TORRES, R.C.S.; ARAÚJO, R.B.; FERREIRA, P.M. et al. Frequência de displasia coxofemoral em cães da raça Rottweiler em Minas Gerais. Arquivo Brasileiro de Medicina Veterinária e Zootecnia. V.51, n.1, p.39-40, 1999a.

TORRES, R.C.S.; FERREIRA, P.M.; ARAUJO, R.B. et al. Presence of Morgan's line as an indicator of canine hip dysplasia in German Shepherd dogs. Arquivo Brasileiro de Medicina Veterinária e Zootecnia, v.51, n.2, p.157-158, 1999b.

TORRES, R.C.S.; ROCHA, B.D.; SILVA, E.F. Frequência de displasia coxofemoral em cães da raça Labrador retriever no Estado de Minas Gerais. Arquivo Brasileiro de Medicina Veterinária e Zootecnia, v.53, n.4, p.445-446, 2001.

URTADO, S.L.R. Contribuição ao estudo da displasia coxofemoral em cães da raça Rottweiller: estudo retrospectivo. 2005. 134p.Dissertação (mestrado em Cirurgia) Universidade de São Paulo, Faculdade de Medicina Veterinária e Zootecnia, São Paulo.

VERHOEVEN, G.; COOPMAN, F.; DUCHATEAU, L. et al. Interobserver agreement in the diagnosis of canine hip dysplasia using the standard ventrodorsal hip-extended radiographic method. Journal Small Animal Practice, v. 48, n.7, p.38793, 2007. 